MANCHESTER

The University of Manchester

\section{Posaconazole tablet therapeutic drug monitoring in patients with chronic pulmonary aspergillosis}

\author{
I. Rodriguez-Goncer, C. Kosmidis, R. Richardson, C.B Moore, M.D Richardson, D.W Denning
}

National Aspergillosis Centre, Manchester University NHS Foundation Trust, Manchester Academic Health Science Centre, University of Manchester, UK

\section{Objectives}

- Posaconazole (POS) tablet offers better bioavailability than liquid suspension and a favorable adverse event $(A E)$ profile.

- Most POS pharmacological data are derived from immunocompromised populations, with little experience in immunocompetent hosts such as those with chronic pulmonary aspergillosis (CPA).

- Our aim was to explore pharmacokinetics and AE profile of POS tablets in a cohort of patients with CPA.

\section{Methods}

- Medical records were analyzed retrospectively.

- Therapeutic drug monitoring (TDM) by bioassay after 2-3 weeks was performed. Dose escalation and reduction was recommended according to local guideline.

- Correlation of POS levels with demographics, prescribed dose and frequency and severity of AE was analyzed (1).

\section{Results}

Seventy-two patients received POS tablets. Fifty (69\%) were male. Sixty-one were started on $300 \mathrm{mg}$ daily and 11 were started on $200 \mathrm{mg}$ daily.

- $49(68 \%)$ patients developed attributable AE; $9(18 \%)$ grade 1 , $22(44 \%)$ grade 2, $17(34 \%)$ grade 3 and $1(<0.1 \%)$ grade 4 .

- Seven (9\%) patients had liver function test (LFT) abnormalities. Most frequent attributable $\mathrm{AE}$ were fatigue (37\%), breathlessness (18\%), nausea (12\%), headache $(8 \%)$, peripheral neuropathy (8\%) and diarrhea (6\%).

- AE were present in $115 / 196(58.7 \%)$ occasions in patients on $300 \mathrm{mg}$ and $45 / 115(39.1 \%)$ in patients on $200 \mathrm{mg}$ a day, ( $p$, $<0.01)$.

- Mean POS level was $1.94 \mathrm{mg} / \mathrm{L}$, (range 0.1-6.4).

- Figure 1 shows boxplots of levels with various dosing regimens. For patients on $300 \mathrm{mg}$, mean level was $2.13 \pm 1.13 \mathrm{mg} / \mathrm{L}$, for patients on $200 \mathrm{mg}$, mean was $1.95 \pm 0.84 \mathrm{mg} / \mathrm{L}$ and for patients on $100 \mathrm{mg}$, mean level was $1.08 \pm 0.32 \mathrm{mg} / \mathrm{L}$. Significant differences in levels were: $100 \mathrm{mg}$ vs. $200 \mathrm{mg}(p<0.001), 100 \mathrm{mg}$ vs. $200 / 300 \mathrm{mg}$ ( $p, 0.006)$, $100 \mathrm{mavs} .300 \mathrm{ma}(\mathrm{D}<0.001)$. The difference between $200 \mathrm{ma}$

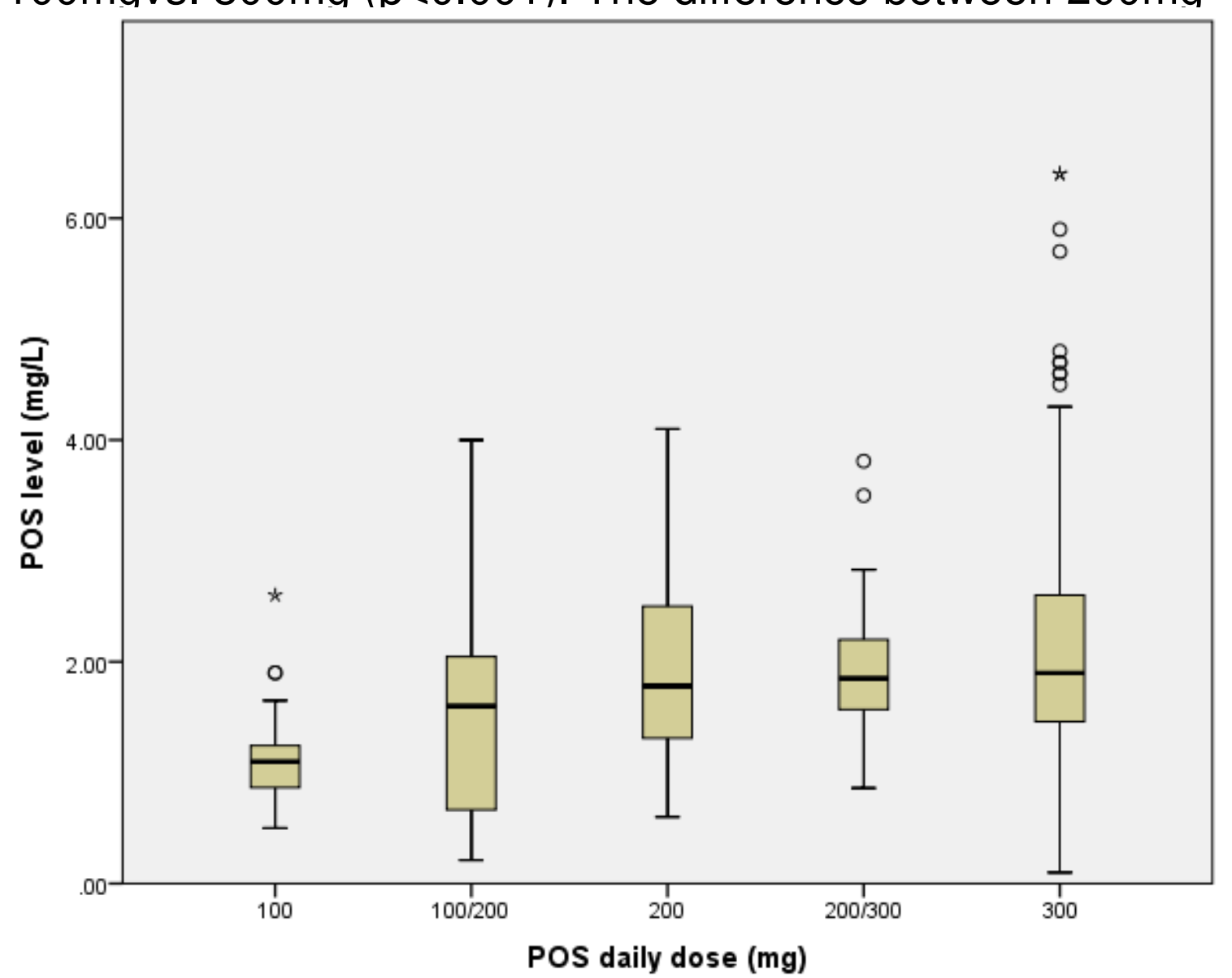

Figure 1. Mean and interquartile range of POS levels according to dosing regimen. (100/200: $100 \mathrm{mg}$ and $200 \mathrm{mg}$ on alternate days; 200/300: $200 \mathrm{mg}$ and $300 \mathrm{mg}$ on alternate days)

\section{Results}

and $300 \mathrm{mg}$ was not significant.

- Mean POS level was $1.81 \pm 0.96$ for patients reporting no $A E$, and $1.90 \pm 1.11$ for those reporting AEs $(p=N S)$. Levels were measured in five patients with LFT elevation: they were 0.8 $\mathrm{mg} / \mathrm{L}, 1.04 \mathrm{mg} / \mathrm{L}, 1.63 \mathrm{mg} / \mathrm{L}, 6.4 \mathrm{mg} / \mathrm{L}$ and $6.4 \mathrm{mg} / \mathrm{L}$.

- Factors associated with AE of Grade 2 or higher were: dose of $200 \mathrm{mg}$ vs $300 \mathrm{mg}$ ( $p, 0.001)$ and asthma (0.008).

- Figure 2 shows intra-patient variability; the mean coefficient of variance was 0.25 (range, 0.12-0.46).

- Table 1 shows risk factors for sub-therapeutic levels.

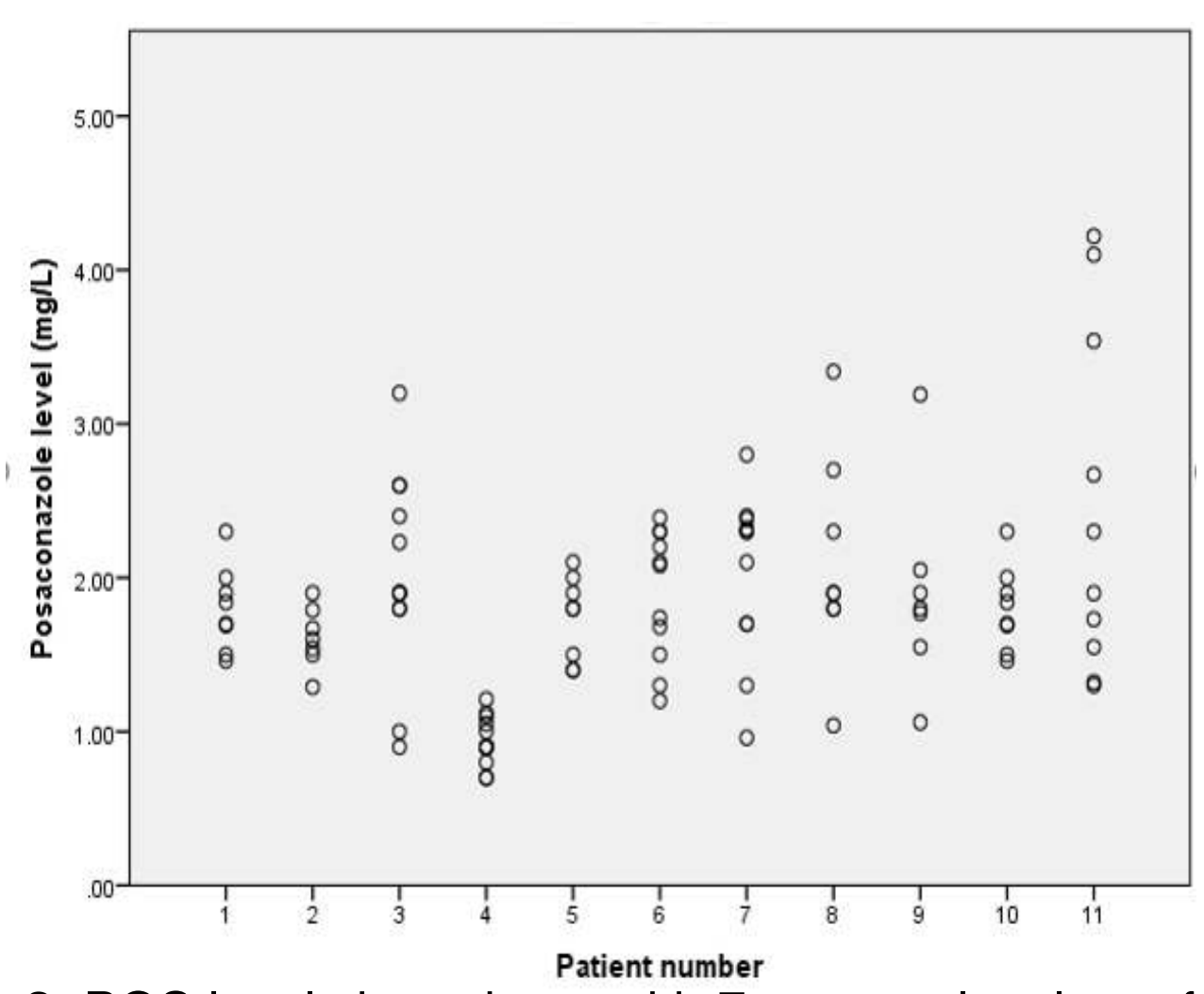

Figure 2. POS levels in patients with 7 or more levels performed on $300 \mathrm{mg}$ per day

Table 1. Risk factors resulting in a sub-therapeutic POS level

\begin{tabular}{|c|c|c|c|c|}
\hline Factor & $<1 \mathrm{mg} / \mathrm{L}$ & $>1 \mathrm{mg} / \mathrm{L}$ & $\begin{array}{l}\mathbf{p} \text { (uni } \\
\text { variate) }\end{array}$ & $\begin{array}{l}\mathbf{p} \text { (multi } \\
\text { variate) }\end{array}$ \\
\hline $\begin{array}{l}\text { Dose (mg) } \\
300 \\
300 / 200 \\
200 \\
200 / 100 \\
100\end{array}$ & $\begin{array}{l}19 / 189(10.1) \\
2 / 30(6.7) \\
11 / 114(9.6) \\
3 / 8(37.5) \\
15 / 43(34.9)\end{array}$ & $\begin{array}{l}170 / 189(89.9) \\
28 / 30(93.3) \\
103 / 114(90.4) \\
5 / 8(62.5) \\
28 / 43(65.1)\end{array}$ & $\begin{array}{l}\text { Ref } \\
0.561 \\
0.909 \\
0.029 \\
<0.001\end{array}$ & $\begin{array}{l}\text { Ref } \\
0.927 \\
0.488 \\
0.053 \\
<0.001\end{array}$ \\
\hline $\begin{array}{l}\text { Age } \\
\text { (years } \pm S D)\end{array}$ & $59.88 \pm 12.1$ & $59.19 \pm 11.4$ & 0.69 & \\
\hline $\begin{array}{l}\text { Weight } \\
\text { (Kg } \pm S D))\end{array}$ & $61.52 \pm 14.75$ & $67.61 \pm 14.95$ & 0.007 & 0.943 \\
\hline $\begin{array}{l}\text { Sex } \\
\text { Male } \\
\text { Female }\end{array}$ & $\begin{array}{l}27(9.8) \\
23(21.1)\end{array}$ & $\begin{array}{l}248(90.2) \\
86(78.9)\end{array}$ & 0.004 & 0.041 \\
\hline $\begin{array}{l}\text { Underlying } \\
\text { condition } \\
\text { COPD } \\
\text { TB } \\
\text { Asthma } \\
\text { Bronchiectasis }\end{array}$ & $\begin{array}{l}17 / 137(12.4) \\
9 / 67(13.4) \\
12 / 47(25.5) \\
3 / 73(4.1)\end{array}$ & $\begin{array}{l}120 / 137(87.6) \\
58 / 67(86.6) \\
35 / 47(74.5) \\
70 / 73(95.9)\end{array}$ & $\begin{array}{l}0.875 \\
0.844 \\
0.011 \\
0.011\end{array}$ & $\begin{array}{l}0.01 \\
0.001\end{array}$ \\
\hline $\begin{array}{l}\text { PPI use } \\
\text { Yes } \\
\text { No }\end{array}$ & $\begin{array}{l}18 / 122(14.8) \\
32 / 262(12.2)\end{array}$ & $\begin{array}{l}104 / 122(85.2) \\
230 / 262(87.8)\end{array}$ & 0.516 & \\
\hline
\end{tabular}

- A daily dose of $200 \mathrm{mg}$ achieved equivalent POS levels to the $300 \mathrm{mg}$ dose and was better tolerated.

- Males were more likely to achieve therapeutic level.

- The underlying condition may have an effect on the level, possibly as a result of co-medication.

- Patients with asthma were more prone to AE. 